\title{
Deploying Public Surface Transit to Forward Messages in DTN
}

\author{
Seilendria A. Hadiwardoyo, Alexandre J. T. Santos \\ Centro Algoritmi \& Dep. Informática, Universidade do Minho \\ Braga, Portugal \\ seilendria.a.h@ieee.org, alex@di.uminho.pt
}

\begin{abstract}
Delay Tolerant Network (DTN) is a communication architecture enabling connectiviety in a topology with unregular end-to-end network connection. DTN enables communication in environments with cross-connectivity, large delays and delivery time variations, and a high error rate. DTN can be used in vehicular networks where public transport get involved. This research aims to analyze the role of public transit as a DTN routing infrastructure. The impact of using public transit as a relay router is investigated by referencing the network performance, defined by its delivery ratio, average delay and overhead. The results show that public transit can be used as a backbone for DTN in an urban scenario using existing protocols. This opens insights for future researches on routing algorithm and protocol design.
\end{abstract}

Keywords-Delay Tolerant Networks, Public Transit, Routing Protocol

\section{INTRODUCTION}

In an urban environment, communication technologies can be used for an intelligent transport system. Named vehicular networks, this type of communication enables vehicles to communicate each other or communicate with Road Side Units (RSU) infrastructures. The benefits that can be taken from this system include providing awareness of events in the traffic or to estimate the travel time. This opens the opportunities to enhance the safety among the drivers or providing other commercial applications like entertainment by reducing cost without using cell services. This type of technology is now being developed and aims to deliver a good quality of service by considering several layered perspectives of communciation systems [1].

Our interest in this research is using DTN [2] as an alternative solution to send data in a bigger scale to reduce the cost, or even the lack of infrastructures. One interesting issue in DTN is the routing mechanism. DTN deployment key factor is how to find route from one source to its destination, where the end-to-end paths are unstable and varying over time. In this type of network, a source that will send the message will store the bundle if a contact is not available. When a source node is in contact with an intermediate node that is closer to the destination node, it will forward the bundle. Then, the intermediate node will carry the bundle when a contact is not available. Whenever the node has not yet met the destination, the process is repeated and the bundle will be relayed hop by hop or

978-1-4799-5344-8/15/\$31.00 @ 2015 IEEE following the store-carry-forward paradigm as depicted in Figure 1. As we can see from the figure, a node will keep a bundle from source node in a local storage, then based on routing decisions it will also forward the bundle to another chosen relay node that will mimetize this behavior. Once a relay node meets the destination node, the bundle will be delivered to the destination node.

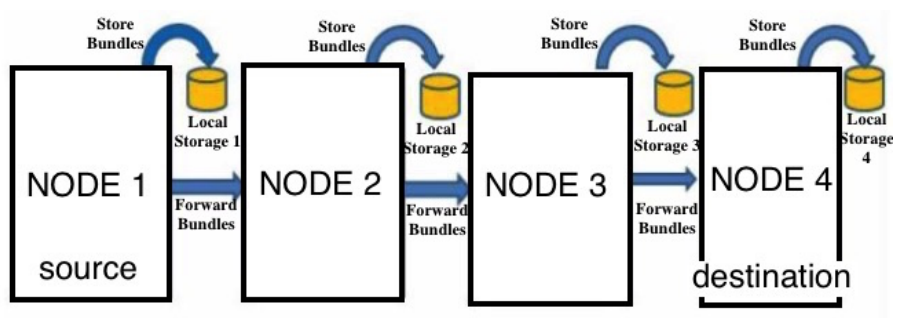

Figure 1: DTN Architecture Scheme

Recent researches have been carried out to overcome the problem of routing in DTN, especially in the field of transport [3]-[5]. Researchers see the advantages of the characteristics of public transport systems. Some specific characteristics, e.g. timetables and network maps can be exploited for routing. DieselNet [6] is one of the initiative researches that exploits the use of buses as a public transport to distribute messages. Using real mobility and real transfer, the network operates in a large geographic area between five colleges using MaxProp [6] as its routing protocol. Inspired by this idea, we aim to implement it in a metropolitan environment where the private vehicles and bus station plays its roles as nodes capable of distributing information.

In order to prove the advantage of using public transport for the communications within an urban environment, we need to test its network performance. However, the implementation in the real world will involve high costs and is too much expensive and the coverage for a wide area cannot possibly be implemented directly. So, using a simulation tool in this research can be a way to study the system. For comparison for this research, we will measure the performance of existing and well known routing protocols that can be studied through simulator softwares like Opportunistic Network Environment Simulator (ONE) [7] and making also use of OpenJump [8].

An ultimate goal is to present, analyze and discuss the drawbacks of known solutions, enabling to characterize the 
behavior of a new routing protocol with better network performance in terms of delivery probability, delay, and network overhead for a city scenario.

The remainder of this paper is organized as follows. Section II introduces the related work on establishing a DTN in a pulic transport environment. Section III presents the simulation scenario and analyzes the obtained results. Section IV concludes the paper and provides guidelines for future work.

\section{RELATED WORKS}

Providing a low-cost communication is primarily an ultimate goal of the DTN concept. Using means of transportation modes like buses or cars, information is carried and distributed. In rural areas of developing countries, this paradigm can be used where Internet connection is rarely found. KioskNet [9] is one of the solution in its kind which was proposed by using a kiosk as an access point. In this scenario, compare to a metropolitan environment, which is our study case in this research, a traffic congestion does not really exist and the density of nodes is not high when we talk about whether the private vehicles or public transport vehicles.

Recently, an Idea of integrating DTN concept within a metropolitan transportation system called MetroNet [10] has been proposed. MetroNet exploits the precise schedules of metro systems for providing Internet downloads even if an end-to-end path between the source and the destination node does not exist contemporaneously. However, the authors stated that their research is not focussed on routing solution as they assume that trains only interact with onehop stations.

Several efforts have been done in developing a routing protocol for an implementation in an urban public transportation system. Using bus as relay for data delivery can be an option [11]. More specifically, a DTN routing algorithm which exploits the characteristics of urban transportation systems can have low resource utilization, low latency, and high delivery rates [3]. However, the routing protocol [3] is deployed in very special scenarios, in which the public transport respects the timetable.

A position-based routing protocol for metropolitan bus networks was proposed [12]. It makes use of street map and bus route information to identify a stable geographic route. Another approach uses less number of hops, using line hop as an alternative to have lower network load. A minimum distance between bus lines are taken as consideration. [13]. Based on the bus lifetime or the connection time between bus is also another way to develop a single path routing protocol [14]. The inter-contact times between public transport vehicles can be estimated through exponential calculation, assuming that the vehicle nodes act as a backbone [15]. For an efficient transmission path to avoid broadcast storm, a footmark leaving scheme can be used as well in a bus-based network [16]. A better delivery probability can also be achieved by giving a deadline for each packet so that a single-copy routing can be used as an alternative [17]. A realistic bus delay tolerant network has a DTN routing with a packet having a deadline relaying on the traces of the schedule of a large public transportation system so that under a stochastic model it maximize the delivery probability for bus arrivals at stops [17]. Polarity can also be used in a position based routing to forward packets according to the location of the destination [18]. Others work using public bus vehicles for a DTN communications has been recently developed as well [5], [19]

However, we should also take into account the influence of mobile node density and mobility models for the performance of DTN [20]. A mobility model can help producing inter-contact time and contact time distributions that follow closely the ones found in the traces from the realworld measurement experiments [21]. Mobility model also affects the frequency and duration of opportunities for data transfer between nodes, which is a key factor of network performance, so whether we can choose a model according to realistic scenes is important for the accuracy and practicability of simulation. Inter-contact time, contact time distribution and other contact information can be used to determine routing protocols [22]. Exponentiality of node inter-arrival times also shows how node mobility information can be used to obtain accurate analysis of important performance metrics under DTN routing schemes [23]. Choosing the right level of model granularity should be done when modeling mobility-related measures such as inter-contact times in DTN [24].

\section{DTN Routing Protocols Simulation}

Considering these existing works, a DTN scenario within public transport environment still deserves research efforts. Thus, for a start, a test of implementing DTN in a city environment where a public transport system is deployed should be done. The DTN is a communication paradigm that takes into account the fact that the connection occurred in a limited of time. In this case, as the node moves dynamically in a certain period of time and velocity, the connection should be taken as fast as possible.

As our goal is to compare an opportunistic network scheme in a city environment, it is needed to implement an appropriate model for the movement of the nodes in the environment. One should produce the contact information from real-world measurements. For better network performance, frequency and duration of probable node encounters data transfer between nodes, a suitable mobility model should be defined. Thus, it will help to compare the existing protocols, such as MaxProp and Spray and Wait.

In order to evaluate DTN routing protocols characteristics in a urban scenario, making use of BRT public transport systems, we have established a scenario based upon a region within the city of Jakarta, also making use of real BRT public transport lanes existing in that area.

The scenario to be implemented in the simulation in order to evaluate the network will now be presented. Thus, we have to analyze the characteristics of the vehicular components and the network parameters used in the scenario. 


\section{A. Characteristics of Public Transport Systems}

In this research, we used urban public transport systems scenario in which the vehicles concerned are operating on surface infrastructure. In such transport systems, buses that run on roads can be a backbone for DTN. The public buses has lines and stops. The movement of buses respects the traject, which define the route or the predefined movement of buses. Buses has timetable as well to define the arrival and departure time and the frequency of the service. According the number of passengers who benefit this service, the frequency of buses change over a given time. Usually there is a difference between the service during rush-hour and late evening. The frequency of buses tends to be stable between the working hours, precisely between 9 AM until 3 PM on weekdays as seen on Figure 3 . The movement pattern does not change between these 6 hours period [25], [26].

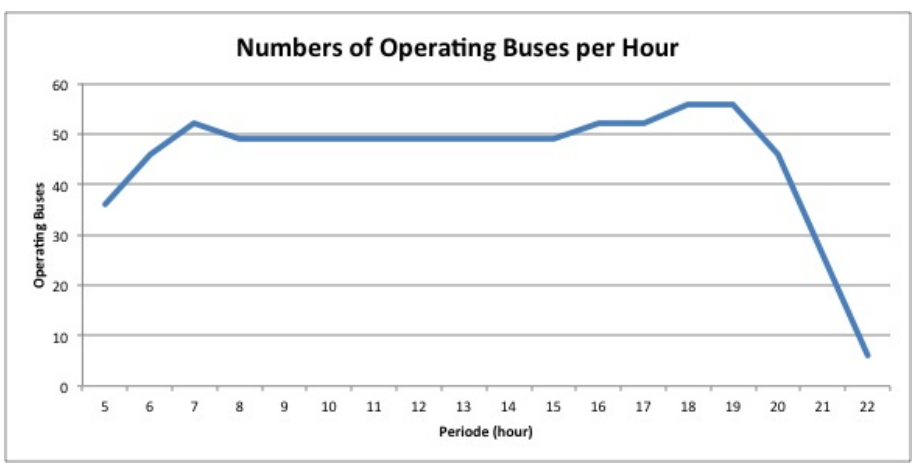

Figure 2: Operating Buses per hour of Transjakarta's BRT

Bus Rapid Transit has more flexibility and reliability than other regular bus services. It has a dedicated lane in the road where it shares with other private vehicles. Having this previlege, BRT has a more stable movement in terms of speed and more likely to meet the schedule requirements. In this research we are interested in investigating the BRT in Jakarta, specifically in main roads where a business district is located.

We will try to see the benefit of using BRT vehicles as relay nodes to forward DTN packets in a city environment. When two buses are in radio range, a communication can be achieved. Buses where the movement can be predicted will have a high probability of contact compare to private vehicles. Also, buses have better technical properties than regular private vehicles, closer to the properties owned by fixed infrastructures or RSUs. By looking the capabilities of BRT, we can provide a network for information distribution in a city environment that is extensible and less costly.

\section{B. Simulation Setup}

A realistic scenario is desired in building an urban network of communications. We would like to use the example of the city of Jakarta in Indonesia as a scenario. First, a cartographical material from OpenStreetMap of the city area of Jakarta will be exported to OpenJump
[8]. The sample area we are using is the busiest district in Jakarta. Sudirman, dubbed as the central business district of Jakarta where governmental offices and corporate headquarters are located, is taken as the experimental location for the network Figure 2. The size of the area is $4.5 \mathrm{~km}$ height and $3.4 \mathrm{~km}$ width.

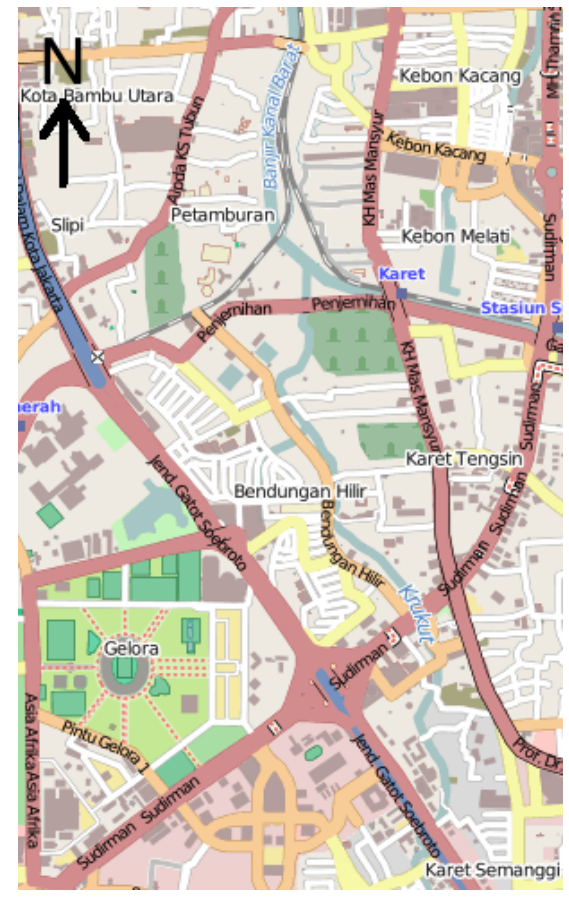

Figure 3: Sample Map of Jakarta

In order to get a map for a map-based movement, we used OpenJump to draw a cartographic map. OpenJump is a Geographical Information System (GIS) software that enables us to draw the routes for the vehicles movement. OpenJump is a Java based vector and open source written in the Java programming language. By using OpenJump, we can create a cartographic map for movements of nodes in a WKT format that may easily be imported to ONE simulator. Thus, it allows us to build trajectories or paths for nodes to move in the networks.

ONE simulator [7] is used to evaluate the efficiency of the method. The simulator that has been written in Java and open source. It is devoted for delay tolerant networks. This tool is used mostly by the DTN research community for routing protocols. In which we can simulate different mobility models for specific scenarios.

The simulaton is writen in Java which is configurable for simulating the behaviours of nodes, such as movement and connections. A Graphical User Interface (GUI) is included in this simulator for a visual feedback. Routing protocols can also be designed in this simulator with routing modules configured.

Depending on the simulator settings, appropriate movement models and routing protocols can be implemented in an independent module. Easy implementation of new mobility and routing protocols is possible using this simulator. Network performance of completed simulations 
is reported in the report files which are created using modules dynamically loaded with movement and routing modules.

In this research scenario, we deploy 100 nodes into 2 groups in the network, consisting of private vehicles and BRT buses. The scenario has 2 BRT lines, where the velocity of buses varies between $22 \mathrm{~km} / \mathrm{h}$ and $72 \mathrm{~km} / \mathrm{h}$. In the other hand, private vehicles run between $11 \mathrm{~km} / \mathrm{h}$ and $40 \mathrm{~km} / \mathrm{h}$. The simulation is run for 6 hours, taking example of a stable vehicle movement at 9 AM until 3 PM. The private vehicles will follow the Shortest Path Map Based Movement (SPMBM) and the buses will follow the Map Route Movement (MRM)

\section{TABLE I: Simulation Parameters}

\begin{tabular}{|l|l|}
\hline \multicolumn{1}{|c|}{ Parameters } & \multicolumn{1}{c|}{ Values } \\
\hline Scenario Time & 12 Hours (from 9 AM to 3 PM) \\
\hline Interface & IEEE $802.116 \mathrm{mbps}$ \\
\hline Transmission Range & $30 \mathrm{~m}$ \\
\hline Vehicle Speed & $11 \mathrm{~km} / \mathrm{h}$ to $40 \mathrm{~km} / \mathrm{h}$ \\
\hline Bus Speed & $22 \mathrm{~km} / \mathrm{h}$ to $78 \mathrm{~km} / \mathrm{h}$ \\
\hline Number of Nodes & 100 \\
\hline Message Size & $500 \mathrm{kB}, 1 \mathrm{MB}, 2 \mathrm{MB}$ \\
\hline Movement Models & SPMBM, MRM \\
\hline
\end{tabular}

The interface used for the nodes is using the standard IEEE 802.11 with a 6 Mbps data rate and $30 \mathrm{~m}$ transmission range. Buses have higher data rate, transmission range, and larger buffer space than private vehicles. Vehicles in these two groups can communicate each other whenever they are in range.

Five existing popular routing protocols will be tested in this scenario. First, Direct Delivery Routing [27], a single-copy routing protocols where only one copy of each message exists in the network. First contact [28] is also a single-copy routing protocols. In this protocol, messages are forwarded to the first node they encounter, then the former deletes the message. For a multi-copy routing, Epidemic Routing [29] that performs flooding is an example. It replicate messages to all encountered peers. Spray and Wait [30] routing has a more controlled flooding method. It limits the number of message copies created. The number of copies are predetermined for replication. Last but not least, MaxProp [6] is a routing protocol that takes the city environment as a consideration. Its delivery is based on the high delivery prediction and high priority using city buses as nodes that have high probability to meet again.

Also, we will vary bundle size, with $500 \mathrm{kB}, 1 \mathrm{MB}$ and 2 MB. Three simulation scenarios are evaluated in this paper. The first one is where the network has no BRT service. The ratio rate of private and public transport is $0: 1$. The second one is where we deploy the BRT service in the network. 30 nodes are mounted for the scenario, resulting the ratio to be $0.7: 0.3$. And the last one is where the number of BRT buses is the same as the number of private vehicles, 50 buses and 50 cars or 0.5:0.5.

The network performance is then evaluated by the delivery ratio of delivered bundles in the network, the latency average, and the overhead ratio. This will define whether the flow of DTN packets in the network is delivered reliably, quickly, and efficiently.

\section{Results And Discussion}

As we have set up the simulation, we can retrieve the network performance for the three scenarios by looking at the delivery probability rate, latency average, and the overhead ratio of the network. In total, 45 simulation runs were done. Three scenarios were analyzed varying the type of nodes, five different routing protocols, and with three different size of bundle messages. Each simulation scenario was run five times (each with a different random seed) and calculate the $95 \%$ confidence interval (CI) for the average values.

\section{A. Delivery Probability}

The delivery probability is the ratio of total number of messages delivered to their destination to total number of created messages at source node. In DTN, the delivery ratio is the most important network performance metrics. However, in this network, a message is rarely lost. Rather the network is unable to deliver messages within amount of time. Thus, we define the delivery ratio as the fraction of generated messages that are correctly delivered to the final destination within a given time period. The delivery probability is described as in Eq. (1):

$$
|M d| \div|M|
$$

where $M d$ is the amount of messages delivered to a destination and $M$ is the number of messages created in the network scenario.

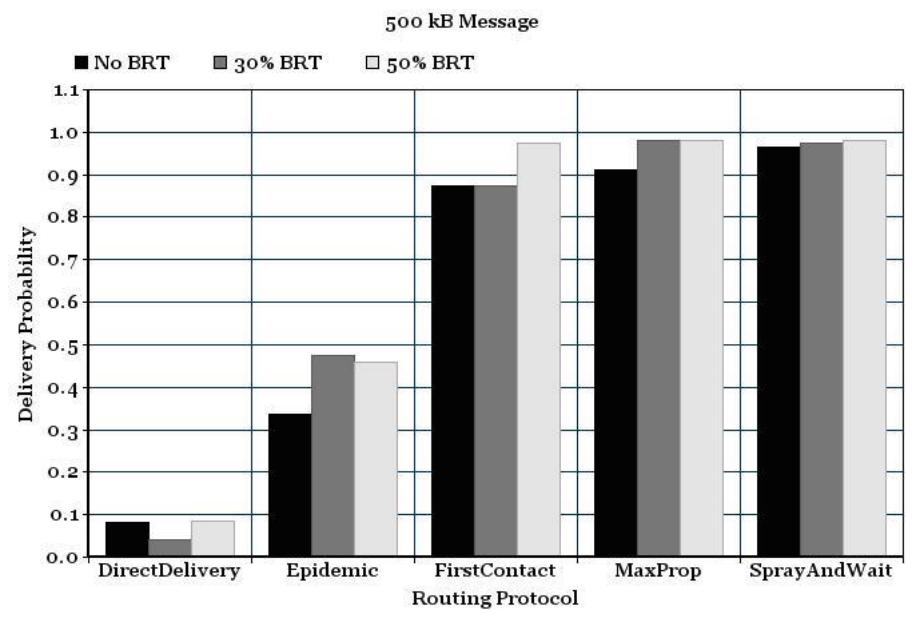

Figure 4: Delivery Ratio for the Three Scenarios with $500 \mathrm{kB}$

As expected, in terms of delivery rate, a scenario where BRT are deployed in the network has better performance. We can see from Figure 4 that as there are more buses in the environment, delivery probability increases. The result is pretty much the same when varying the size of the message as shown in Figure 5 and Figure 6.

\section{B. Latency Average}

Another metric is the latency, the time taken from the instant a message is created and the instant the message 


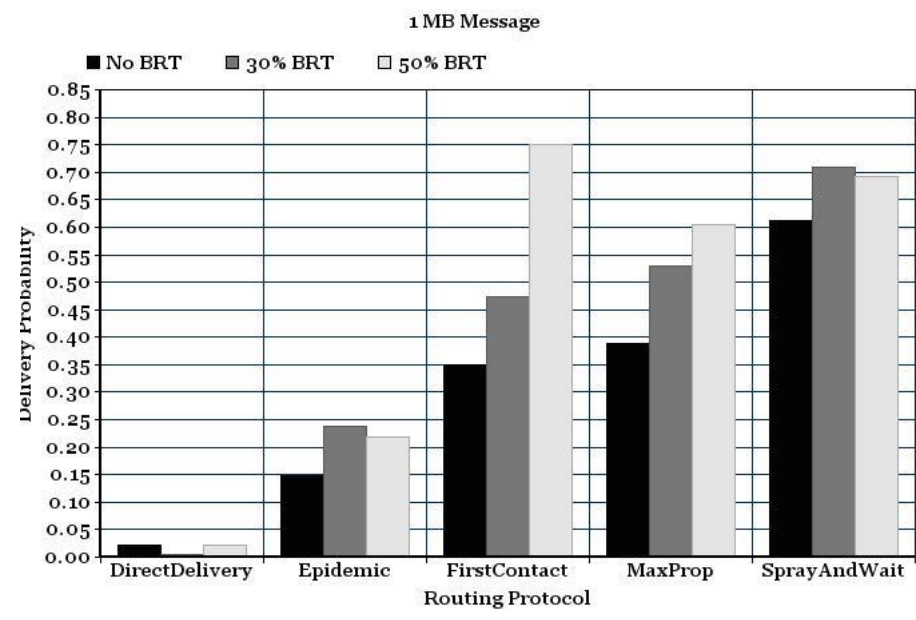

Figure 5: Delivery Ratio for the Three Scenarios with $1 \mathrm{MB}$

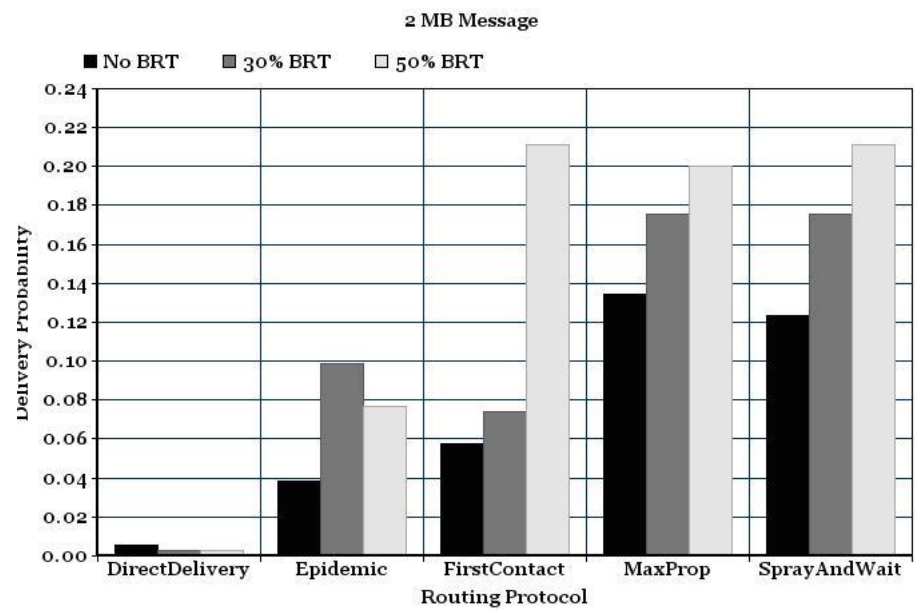

Figure 6: Delivery Ratio for the Three Scenarios with $2 \mathrm{MB}$

is received at a destination. This metric is important since many applications can benefit from a short delivery latency, even though they will tolerate long delays. Many applications also have some time window where the data is useful. For example, if a DTN is used to deliver e-mail to a mobile user, the messages must be delivered before the user moves out of the network. The average message delivery latency is described as in Eq. (2):

$$
\left(\sum_{i=1}^{|M d|}(d i-c i)\right) \div(|M d|)
$$

where $d i$ is the time when the message is delivered and $c i$ is the time when the message is created.

In terms of latency average, scenarios where BRT are deployed are still having better performance. As the ratio of buses in the network increases, the time needed for the message to be delivered is reduced. This shows that by

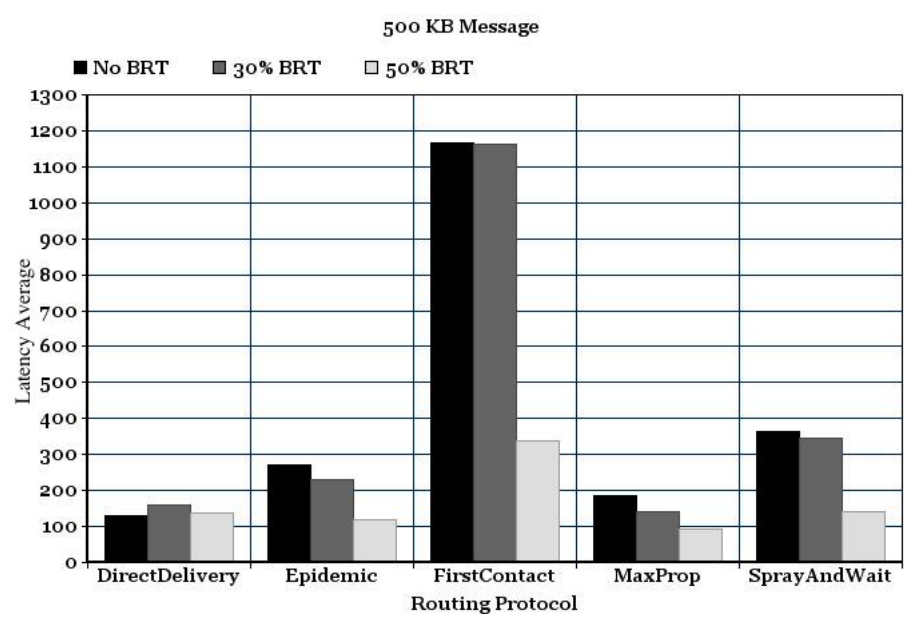

Figure 7: Latency Average for the Three Scenarios with $500 \mathrm{kB}$

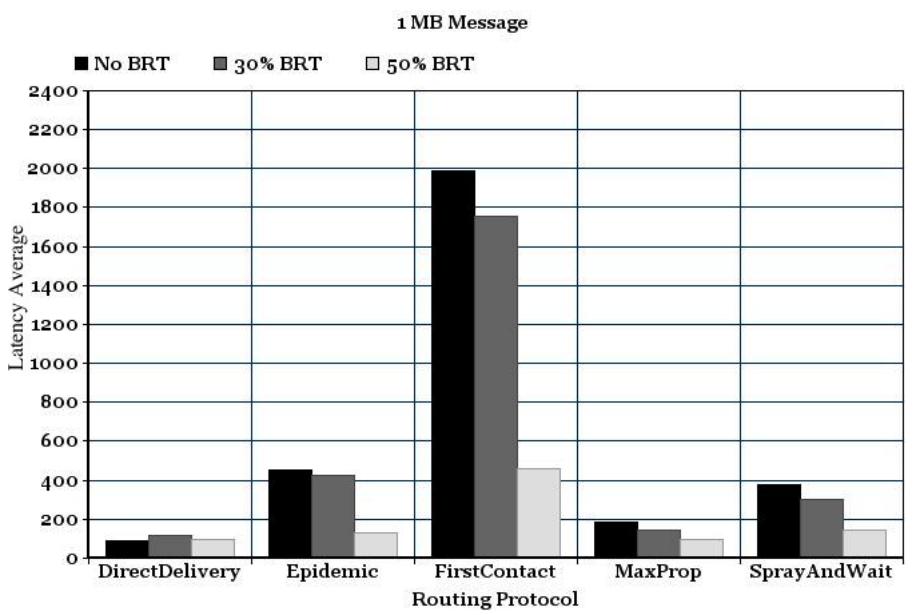

Figure 8: Latency Average for the Three Scenarios with $1 \mathrm{MB}$

adding buses to the network, the delivery of packets will be faster (as shown in Figure 7, Figure 8, and Figure 9).

\section{Overhead Ratio}

The overhead ratio is described as: The amount of messages are transmitted variously in some routing strategies, either because they use multiple copies of each message, make different decisions about the next hop, or because of protocol overhead. The number of transmissions is a measure of the amount of contact capacity consumed by a protocol. It is also an approximate measure of the computational resources required, as there is some processing required for each message. Additionally, each transmission consumes energy, so it is also an approximate measure of power consumption. In this case an overhead ratio will define the power consumption. The overhead ratio reflects the number of redundant packets that are relayed to deliver a single packet. It simply reflects transmission cost in a network. The overhead ratio is described as in Eq. (3): 


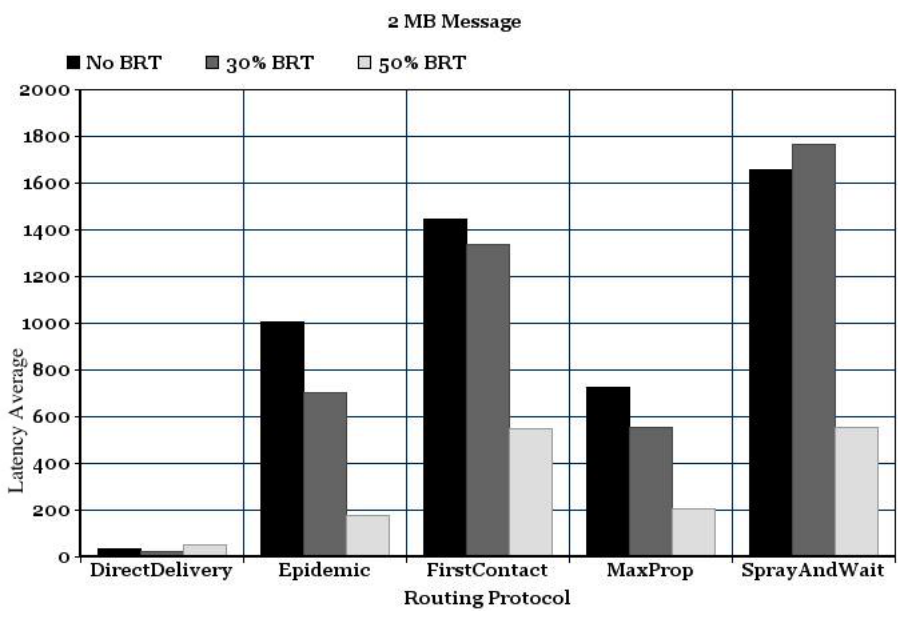

Figure 9: Latency Average for the Three Scenarios with $2 \mathrm{MB}$

$$
\left(\sum_{i=1}^{|M|} r i-|M d|\right) \div|M d|
$$

where $r i$ is the replication of messages created on the network.

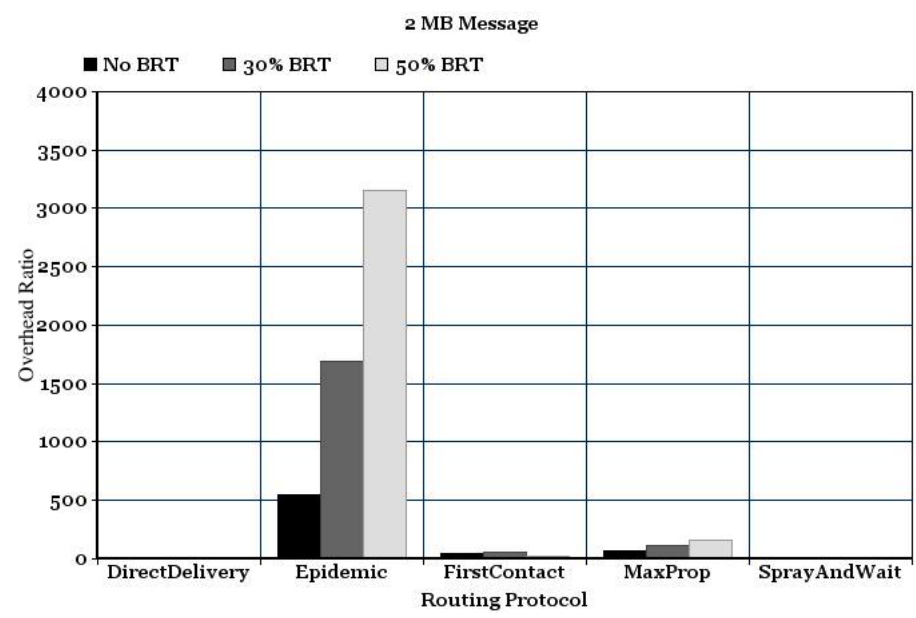

Figure 10: Overhead Ratio for the Three Scenarios with $500 \mathrm{kB}$

Contrary to the other two parameters, in terms of overhead ratio, deploying buses in the network might not be the best idea when using the existing routing protocols. However, in Spray and Wait Routing, the case where BRT is deployed shows better performance as it has less overhead. By varying the size of the message in the network, the result does not change significantly (as shown in Figure 10, Figure 11, and Figure 12). This shows that existing routing protocols used in this study cannot help to provide efficient delivery of message when using public transport means.

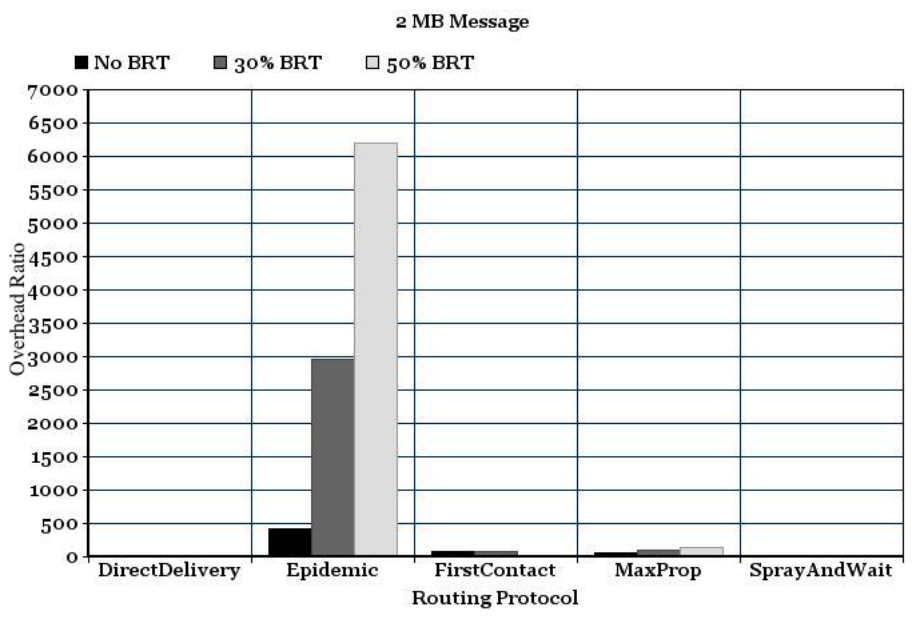

Figure 11: Overhead Ratio for the Three Scenarios with $1 \mathrm{MB}$

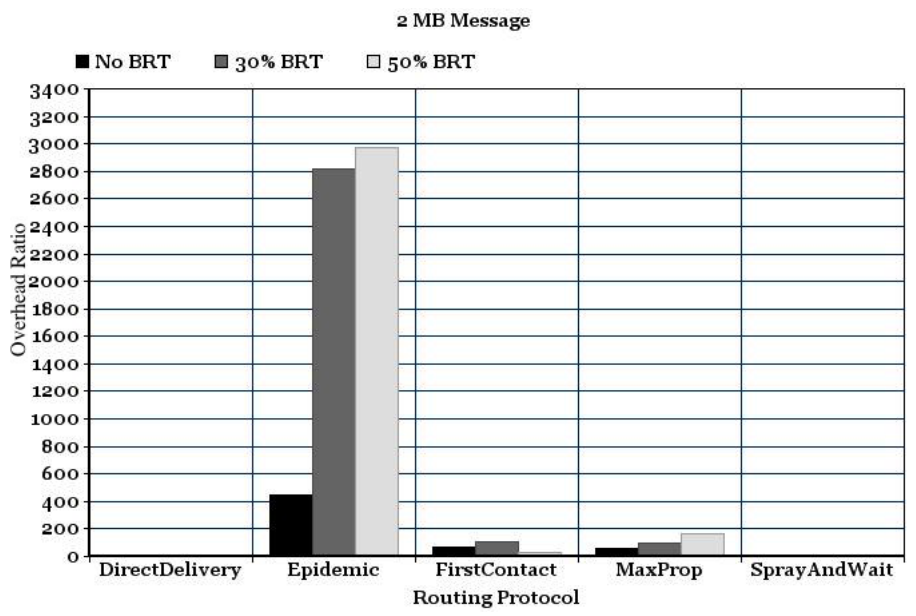

Figure 12: Overhead Ratio for the Three Scenarios with $2 \mathrm{MB}$

\section{Conclusion and Future Works}

In a city scenario, DTN paradigm associated with BRT vehicular nodes can be used to distribute information considering the low cost deployment of communications infrastructures. The predictability of vehicles' movement of public transportation systems can be useful to support the forwarding of messages in vehicular networks. The results obtained from the different simulation scenarios that have been experimented showed that using BRT as a routing infrastructure will help the network to be more reliable and presenting faster flow of message using the existing protocols. The more buses are deployed as a DTN routing nodes, the better network performance will result. However, in terms of duplicated message inside the network, using BRT still generates a considerable overhead. This shows that there is a need for a routing protocol that meets the three criteria in order to establish a better performing network.

Furthermore, by taking advantage of BRT lines in an 
urban scenario, a particular routing algorithm can be defined. Using this strategy, a routing protocol resulted from this research has a good performance in terms of delivery ratio and overhead comparing to the traditional routing protocols. In the future, we aim to propose an alternative routing protocol able to provide a more efficient delivery of messages and adding other means of transportation to turn the network model much closer to the real world. Another concern is to reduce the tradeoff resulted from the protocol. Existing protocols analysed in this research do still present an important drawback in terms of the overhead ratio. We will take into account this parameter in order to have a more efficient routing protocol.

\section{ACKNOWLEDGEMENTS}

This work has been partially supported by FCT Fundação para a Ciência e Tecnologia in the scope of the project: UID/CEC/00319/2013.

\section{REFERENCES}

[1] S. A. Hadiwardoyo, "An overview of qos enhancements for wireless vehicular networks," Network and Complex Systems, vol. 5, no. 1, pp. 22-27, 2015.

[2] K. Fall, "A delay-tolerant network architecture for challenged internets," in Proceedings of the 2003 conference on Applications, technologies, architectures, and protocols for computer communications. ACM, 2003, pp. 27-34.

[3] M. Doering, T. Pögel, and L. Wolf, "Dtn routing in urban public transport systems," in Proceedings of the 5th ACM workshop on Challenged networks. ACM, 2010, pp. 55-62.

[4] T. Pögel, "Optimized dtn-routing for urban public transport systems," in OASIcs-OpenAccess Series in Informatics, vol. 17. Schloss Dagstuhl-Leibniz-Zentrum fuer Informatik, 2011.

[5] X. Ma, X. Zhan, Z. Ouyang, and J. Bai, "Dtns routing protocol based on urban public transport system," in 2nd International Conference on Soft Computing in Information Communication Technology. Atlantis Press, 2014.

[6] J. Burgess, B. Gallagher, D. Jensen, and B. N. Levine, "Maxprop: Routing for vehicle-based disruption-tolerant networks." in INFOCOM, vol. 6, 2006, pp. 1-11.

[7] A. Keränen, J. Ott, and T. Kärkkäinen, "The one simulator for dtn protocol evaluation," in Proceedings of the 2nd international conference on simulation tools and techniques. ICST (Institute for Computer Sciences, Social-Informatics and Telecommunications Engineering), 2009, p. 55.

[8] JUMP, OpenJUMP GIS - the free, java-based open source GIS, 2008. [Online]. Available: http://www.openjump.org

[9] A. Seth, D. Kroeker, M. Zaharia, S. Guo, and S. Keshav, "Lowcost communication for rural internet kiosks using mechanical backhaul," in Proceedings of the 12th annual international conference on Mobile computing and networking. ACM, 2006, pp. 334-345.

[10] L.-P. Tung, Y.-C. Chen, K.-c. Lan, and L.-J. Chen, "Metronet: a disruption-tolerant approach for mobile downloads on metro systems," Transactions on Emerging Telecommunications Technologies, vol. 25, no. 8, pp. 835-851, 2014.

[11] C. Li, L. Zhijun, and J. Shouxu, "An efficient routing protocol in a bus-based vehicular ad hoc network," in Pervasive Computing Signal Processing and Applications (PCSPA), 2010 First International Conference on. IEEE, 2010, pp. 70-73.

[12] T. Li, S. K. Hazra, and W. Seah, "A position-based routing protocol for metropolitan bus networks," in Vehicular Technology Conference, 2005. VTC 2005-Spring. 2005 IEEE 61st, vol. 4. IEEE, 2005, pp. 2315-2319.
[13] M. Sede, X. Li, D. Li, M.-Y. Wu, M. Li, and W. Shu, "Routing in large-scale buses ad hoc networks," in Wireless Communications and Networking Conference, 2008. WCNC 2008. IEEE. IEEE, 2008, pp. 2711-2716.

[14] L. Kuang, M. Xu, and Z. Wang, "An adaptive routing protocol for bus networks," in Advanced Information Networking and Applications (AINA), 2010 24th IEEE International Conference on. IEEE, 2010, pp. 98-104.

[15] L. Hartog, T. Spyropoulos, and F. Legendre, "Using public transportation as a dtn backbone: Mobility properties and performance analysis," Proceedings of AOC, vol. 2010, 2010

[16] W. K. Lai, K.-T. Yang, and M.-C. Li, "Bus assisted connectionless routing protocol for metropolitan vanet," in Genetic and Evolutionary Computing (ICGEC), 2011 Fifth International Conference on. IEEE, 2011, pp. 57-60.

[17] U. G. Acer, P. Giaccone, D. Hay, G. Neglia, and S. Tarapiah, "Timely data delivery in a realistic bus network," Vehicular Technology, IEEE Transactions on, vol. 61, no. 3, pp. 12511265,2012

[18] H.-S. Park, J.-H. Jang, S.-H. Lee, and J.-D. Kim, "Positionbased DTN routing in metropolitan bus network," in Systems and Informatics (ICSAI), 2012 International Conference on. IEEE, 2012, pp. 1449-1453.

[19] T. Shamspour, S. Yousefi, and J. Bagherzadeh, "Performance improvement of vehicular delay tolerant networks using public transportation systems," International Journal, 2013.

[20] V. N. Soares, F. Farahmand, and J. J. P. Rodrigues, "Impact of vehicle movement models on vdtn routing strategies for rural connectivity," International Journal of Mobile Network Design and Innovation, vol. 3, no. 2, pp. 103-111, 2009.

[21] F. Ekman, A. Keränen, J. Karvo, and J. Ott, "Working day movement model," in Proceedings of the 1st ACM SIGMOBILE workshop on Mobility models. ACM, 2008, pp. 33-40.

[22] X. Zhu, Y. Bai, W. Yang, Y. Peng, and C. Bi, "Same: A students' daily activity mobility model for campus delaytolerant networks," in Communications (APCC), 2012 18th Asia-Pacific Conference on. IEEE, 2012, pp. 528-533.

[23] M. Abdulla and R. Simon, "The impact of the mobility model on delay tolerant networking performance analysis," in Simulation Symposium, 2007. ANSS'07. 40th Annual. IEEE, 2007, pp. 177-184.

[24] X. Zhang, J. Kurose, B. N. Levine, D. Towsley, and H. Zhang, "Study of a bus-based disruption-tolerant network: mobility modeling and impact on routing," in Proceedings of the 13th annual ACM international conference on Mobile computing and networking. ACM, 2007, pp. 195-206.

[25] F. R. M. Nur, "Implementation of intelligent autonomous vehicle in public transport: Study case corridor i transjakarta of jakarta - indonesia," Master's thesis, Universitas Indonesia, 2012.

[26] TransJakarta, "Transjakarta website," Accessed on January 2015. [Online]. Available: http://www.transjakarta.co.id/

[27] T. Spyropoulos, K. Psounis, and C. S. Raghavendra, "Singlecopy routing in intermittently connected mobile networks," in Sensor and Ad Hoc Communications and Networks, 2004. IEEE SECON 2004. 2004 First Annual IEEE Communications Society Conference on. IEEE, 2004, pp. 235-244.

[28] S. Jain, K. Fall, and R. Patra, Routing in a delay tolerant network. ACM, 2004, vol. 34, no. 4.

[29] A. Vahdat, D. Becker et al., "Epidemic routing for partially connected ad hoc networks," Technical Report CS-200006, Duke University, Tech. Rep., 2000.

[30] T. Spyropoulos, K. Psounis, and C. S. Raghavendra, "Spray and wait: an efficient routing scheme for intermittently connected mobile networks," in Proceedings of the 2005 ACM SIGCOMM workshop on Delay-tolerant networking. ACM, 2005, pp. 252-259. 